\title{
Vigilancia nutricional en escolares chilenos: Validez de la información
}

\author{
Hugo Amigo ${ }^{1}$, Marcia Erazo $^{1}$, Patricia Bustos ${ }^{1}$, \\ Carolina Aguilar2a, Marcela Taibo ${ }^{3 a}$. \\ Nutritional surveillance \\ in Chilean schoolchildren: \\ validity of the information
}

Background: Anthropometry is used to survey health and nutritional situation of the population. Therefore the quality of the information that is being used must be evaluated. Aim: To estimate the agreement in weight, height and nutritional status in school-children, comparing measurements made by teachers in schools and a standardized and supervised team of professionals. Material and methods: Cross sectional study, including 927 school-children in 31 schools from 7 counties of Santiago. Schools were randomly chosen and the universe of children attending to first grade was measured. Weight, height and nutritional status collected by teachers and researches, were compared. Results: Total agreement for nutritional status reached 0.67, random-weighted Kappa was 0.40 and weighted Kappa, 0.42. Teachers tended to over diagnose under-nutrition and under diagnose overweight and obesity, measuring 270 grams less than the qualified team $(p<0.001)$ and 1.7 $\mathrm{cm}$ more in height $(\mathrm{p}<0.001)$, what is reflected in a difference of less than one point in body mass index $(p<0.001)$. Discrepancies in height and body mass index were higher in extreme values. Conclusions: There is a low agreement between the measurements taken by the research team and teachers. Even though there are discrepancies between measurements, high levels of overweight and low prevalences of stunting and underweight are kept, reflecting problems with exactitude, but not bias. Corrective actions to improve the quality of information, which should include training programs for teachers, instrument maintenance, supervision and verification system for data entry, are needed (Rev Méd Chile 2008; 136: 989-95).

(Key words: Anthropometry; Nutrition assessment; School-aged population)

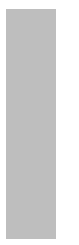

Recibido el 27 de septiembre, 2007. Aceptado el 7 de abril, 2008.

Financiado por la Junta Nacional de Auxilio Escolar y Becas, Ministerio de Educación de Chile.

${ }^{1}$ Departamento de Nutrición, Facultad de Medicina, Universidad de Chile. ${ }^{2}$ Programa

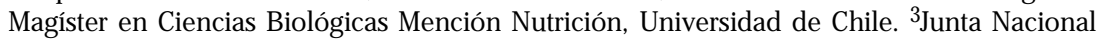
de Auxilio Escolar y Becas.

aNutricionista

Correspondencia a: Dr. Hugo Amigo C. Departamento de Nutrición, Facultad de Medicina, Universidad de Chile. Independencia 1027, Santiago, Chile. Teléfono: 56-2-9786213.

Fax: 56-2-7355581. E mail: hamigo@med.uchile.cl 
L a vigilancia epidemiológica nutricional es útil para monitorear modificaciones del estado nutricional a nivel poblacional. Esta actividad es necesaria, debido a los rápidos cambios que se han observado en varios países del continente ${ }^{1-3} \mathrm{y}$ por las consecuencias sobre el estado de salud de los problemas nutricionales ${ }^{4,5}$.

La vigilancia debería permitir un seguimiento en el tiempo, con el fin de analizar los cambios y tomar decisiones oportunas tendientes a reducir la incidencia y prevalencia de los principales problemas nutricionales. Para hacer este seguimiento es necesario tener indicadores claramente definidos, basados en métodos de recolección de información confiable y de alta validez, para aquello, la obtención del dato debe ser efectuada con personal capacitado y estandarizado, bajo controles de calidad, lo que constituye motivo de preocupación de investigadores y organismos internacionales ${ }^{6-8}$.

En Chile, para evaluar la situación nutricional en los escolares, desde hace más de una década, se ha utilizado información que se genera a nivel de las escuelas en un censo anual de antropometría, cuya información es reunida y analizada en forma centralizada por la Junta Nacional de Auxilio Escolar y Becas (JUNAEB), organismo dependiente del Ministerio de Educación ${ }^{9}$. Por lo tanto, la calidad de la información y la metodología empleada para recolectarla deben requerir de atención para no sobreestimar ni subestimar los resultados.

El objetivo de este estudio fue analizar la calidad de la información que está siendo utilizada para evaluar el estado nutricional de los escolares de la ciudad de Santiago, mediante la comparación de las mediciones antropométricas que anualmente recolectan los profesores y las realizadas por un equipo de profesionales capacitados y siguiendo pautas estandarizadas de medición.

\section{MATERIAL Y MÉTODO}

Estudio de corte transversal de escolares de primer año básico que asistían a escuelas municipales y particulares subvencionadas de siete comunas de la Región Metropolitana de Chile, evaluados al inicio del año escolar 2005. Fueron seleccionadas en forma aleatoria 31 escuelas urbanas de la ciudad de Santiago y al interior de cada escuela se midieron todos los escolares de primer año básico ( $\mathrm{n}=1.057$ niños).

Entre el 7 de marzo y el 13 de abril, los escolares fueron medidos por profesores de los respectivos establecimientos educacionales, siguiendo la norma del Ministerio de Educación ${ }^{10}$, información que fue almacenada en una base de datos a nivel central en la JUNAEB. Los investigadores constataron que la mayoría de los profesores habían recibido capacitación, aunque comprobaron deficiencias en los equipos (balanzas no calibradas y antropómetros sin graduación en milímetros) y en una de las escuelas la información fue enviada por los apoderados según información del consultorio. Desde el 28 de marzo hasta el 28 de abril, con un desfase promedio de 30 días, los mismos niños fueron evaluados por el equipo de investigadores y de éstos, 36,1\% fueron medidos en el mismo mes que lo habían efectuado los profesores.

El equipo de investigadores midió el peso y talla con un mínimo de ropa, utilizando una balanza electrónica digital con antropómetro incorporado (Seca ${ }^{\circledR}$ ), con precisión de $100 \mathrm{~g}$ para el peso y de $1 \mathrm{~mm}$ para la estatura; instrumento que fue calibrado periódicamente. Las mediciones las realizaron profesionales capacitadas de acuerdo a una guía de procedimiento (dos nutricionistas y una enfermera universitaria), previamente supervisadas para minimizar la variabilidad entre observadores y vigilar la precisión y exactitud de las medidas, así como la calibración periódica de los instrumentos de medición de acuerdo a técnicas recomendadas internacionalmente ${ }^{11}$.

El estado nutricional de cada niño se evaluó en función del peso y la talla a través del índice de masa corporal (IMC) según edad y sexo, utilizando como patrón de referencia CDC-NCHS ${ }^{12}$. Los puntos de corte del IMC fueron los siguientes, bajo peso: < percentil 10; peso normal: entre percentil 10 y 85, sobrepeso: entre percentil 85 y 95 y obesidad: $\geq$ percentil 95 .

Ambas medidas antropométricas, la efectuada por profesores y la realizada por el equipo de investigadores, y los diagnósticos nutricionales derivados de ellas fueron reunidas en una base de datos única, utilizando como identificador común el Rol Único Nacional (RUN). Para las compara- 
ciones se utilizó el coeficiente de correlación de Pearson cuando las variables eran continuas (peso, talla e IMC), para evaluar la concordancia entre los diagnósticos nutricionales se utilizó un índice básico de concordancia, que se obtiene sumando las proporciones de mediciones concordantes (la diagonal de la tabla). También se estimó el índice de concordancia Kappa simple de Cohen, que incluye corrección por concordancia debida al azar y el Kappa ponderado de Cohen, que penaliza las discordancias según su magnitud ${ }^{13-15}$.

Para evitar un posible sesgo de subestimación del peso por las diferencias de las fechas de medición (profesores y profesionales vinculados a la Facultad de Medicina), se efectuó una corrección estandarizada del peso incrementando $6,6 \mathrm{~g}$ por día de acuerdo al incremento de peso espera- do para la edad y sexo de los menores localizados en el percentil 50 de la referencia internacional. Posteriormente, en los análisis la muestra fue estratificada en los que habían sido examinados en igual fecha o con una diferencia mínima de 10 días los que habían superado esa diferencia.

Fueron examinados 1.057 niños, pero se logró emparejar 927 casos por falta del identificador (RUN) en la base de datos de JUNAEB. Es importante señalar que los casos perdidos no presentaron un estado nutricional distinto del resto de los niños examinados. En el procesamiento de los datos se verificó la presencia de valores atípicos, que no fueron considerados para los análisis (para definir estos valores se consideró aquellos cuyas diferencias fueron superiores al percentil 950 inferiores al percentil 5 de la distribución de la muestra) la mayoría de ellos

Tabla 1. Nivel de acuerdo en las mediciones de los observadores

\begin{tabular}{|c|c|c|c|c|c|c|}
\hline \multicolumn{7}{|c|}{ Mediciones realizadas } \\
\hline & \multicolumn{2}{|c|}{ Peso (Kg) } & \multicolumn{2}{|c|}{ Talla $(\mathrm{cm})$} & \multicolumn{2}{|c|}{ IMC } \\
\hline & $\begin{array}{c}\text { Equipo } \\
\text { investigador }\end{array}$ & Profesores & $\begin{array}{l}\text { Equipo } \\
\text { investigador }\end{array}$ & Profesores & $\begin{array}{c}\text { Equipo } \\
\text { investigador }\end{array}$ & Profesores \\
\hline Promedio* & $\begin{array}{l}24,91 \\
(0,16)\end{array}$ & $\begin{array}{l}24,64 \\
(0,16)\end{array}$ & $\begin{array}{r}117,67 \\
(0,17)\end{array}$ & $\begin{array}{r}119,38 \\
(0,19)\end{array}$ & $\begin{array}{l}17,89 \\
(0,08)\end{array}$ & $\begin{array}{l}17,22 \\
(0,09)\end{array}$ \\
\hline Diferencias\# & \multicolumn{2}{|c|}{$0,27(0,07)$} & \multicolumn{2}{|c|}{$-1,71(0,09)$} & \multicolumn{2}{|c|}{$0,93(0,05)$} \\
\hline Prueba t & \multicolumn{2}{|c|}{3,61} & \multicolumn{2}{|c|}{$-18,45$} & \multicolumn{2}{|c|}{17,13} \\
\hline Valor de $\mathrm{p}$ & \multicolumn{2}{|c|}{$<0,001$} & \multicolumn{2}{|c|}{$<0,001$} & \multicolumn{2}{|c|}{$<0,001$} \\
\hline
\end{tabular}

*Promedios y su error estándar.

\#Diferencia de los promedios y su error estándar.

Tabla 2. Comparación del diagnóstico nutricional de ambos grupos según IM C

\begin{tabular}{|c|c|c|c|c|c|c|}
\hline & \multicolumn{5}{|c|}{ Profesores } \\
\hline & & Bajo Peso & Eutrófico & Sobrepeso & Obesidad & Total \\
\hline ? & Bajo Peso & 5 & 1 & 0 & 0 & $6(0,8 \%)$ \\
\hline $\begin{array}{c}0 \\
0 \\
0\end{array}$ & Eutrófico & 38 & 280 & 55 & 10 & $383(61,9 \%)$ \\
\hline 8 & Sobrepeso & 1 & 93 & 76 & 25 & $195(24,7 \%)$ \\
\hline : & Obesidad & 0 & 26 & 41 & 137 & $204(25,9 \%)$ \\
\hline 缶 & Total & $44(5,6 \%)$ & $400(50,8 \%)$ & $172(21,8 \%)$ & $172(21,8 \%)$ & $788(100 \%)$ \\
\hline
\end{tabular}

Concordancia total 0,674

Kappa simple: 0,399

$\mathrm{p}=0,000$

Kappa ponderado: 0,427

$\mathrm{p}=0,000$ 
probablemente debido a errores de digitación en la base de datos de la JUNAEB.

Este estudio fue aprobado por el Comité de Ética de la Facultad de Medicina de la Universidad de Chile y los niños fueron medidos después de tener firmado el consentimiento de los padres.

\section{REsultados}

La muestra estuvo constituida en proporciones similares de hombres y mujeres, la mediana de edad de los escolares examinados fue 6 años y 4 meses, sin diferencia significativa por sexo.

$\mathrm{Al}$ analizar las medidas antropométricas obtenidas por ambos grupos (profesores y equipo evaluador) se observó que, en promedio, los profesores obtuvieron valores de peso menores en $270 \mathrm{~g}$ y 1,71 centímetros más de estatura, lo que se refleja en una diferencia de 0,93 puntos de IMC, siendo todas estas diferencias altamente significativas ( $p<0,001$ ) de acuerdo al t-test para muestras pareadas (Tabla 1 ).

Las correlaciones en las medidas obtenidas por ambos equipos fueron altas y significativas, superiores al 0,85 ( $p<0,001$ ) para peso y talla; para IMC fue levemente inferior a $0,80(\mathrm{p}<0,001)$ y se constató que a mayor peso, las discrepancias se concentran en la talla, lo mismo que el IMC en sus valores extremos (Figuras 1, 2 y 3).

La concordancia total para el diagnóstico nutricional según IMC efectuado por los profesores y comparado con el equipo de investigadores, fue levemente inferior al 0,70, siendo que la concordancia corregida por el azar (Kappa simple) fue algo inferior a 0,40 y el índice Kappa ponderado fue algo superior a 0,42. El grupo de profesores tendió a sobreestimar la proporción de niños con bajo peso $(5,6 \%$ vs $0,8 \%$ ) y a subestimar los clasificados como sobrepeso (21,8\% vs $24,7 \%)$ y obesos (21,8 vs 25,9$)$ (Tabla 2). Cuando se estratificó la muestra entre aquellos que fueron evaluados en fechas similares y con más

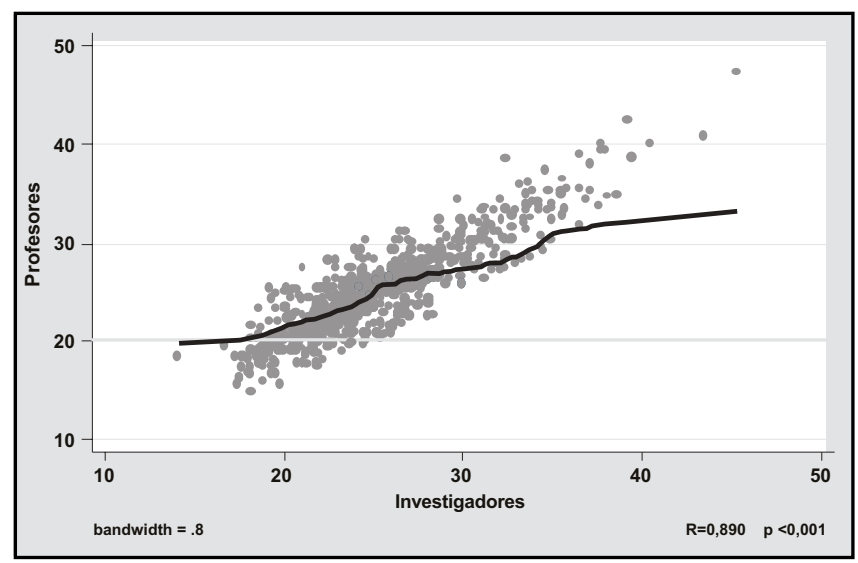

Figura 1. Peso $(\mathrm{Kg})$ según profesores e investigadores.

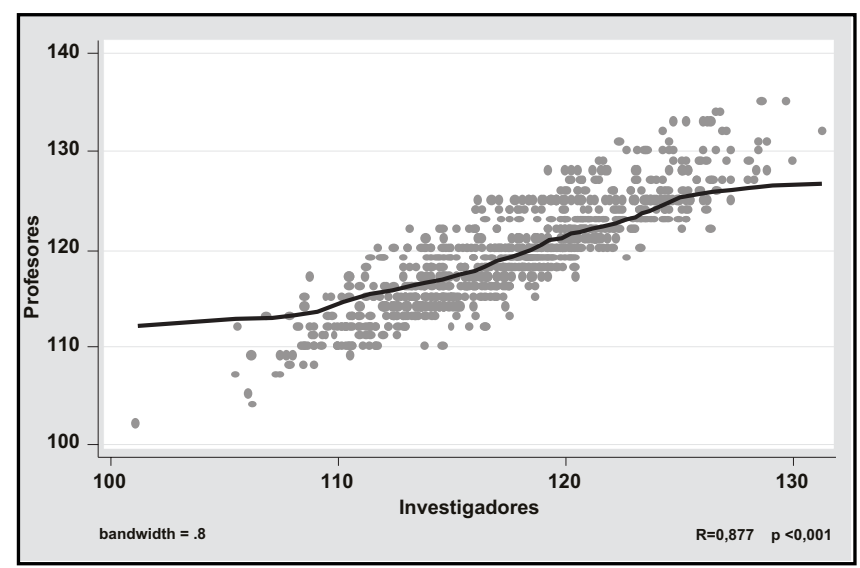

Figura 2. Talla $(\mathrm{cm})$ según profesores e investigadores.

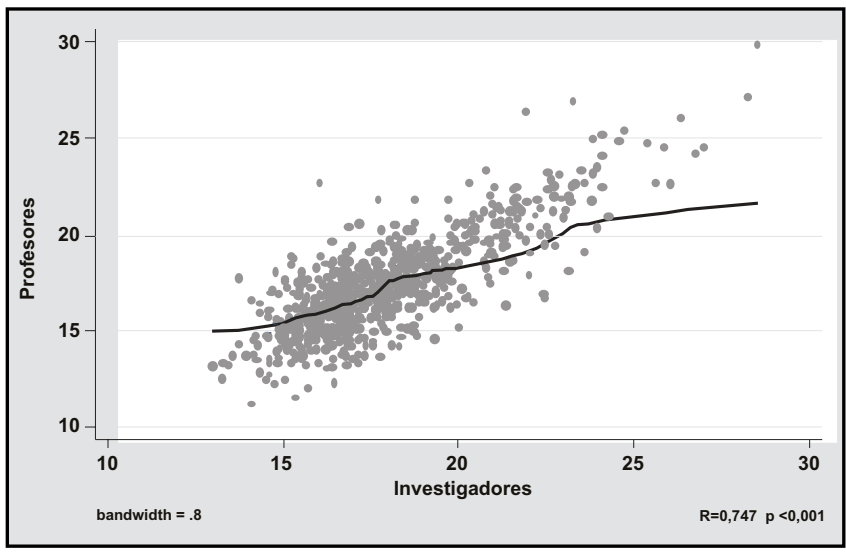

Figura 3. Índice de Masa Corporal según profesores e investigadores. 
de un mes, se observó que los índices Kappa fueron similares, no existiendo diferencias significativas entre ambas evaluaciones.

\section{DISCUSIÓN}

A partir de los resultados de este estudio, se concluye que existe una baja concordancia entre las mediciones realizadas por el equipo evaluador y los profesores de las escuelas. De acuerdo al consenso internacional, ésta puede considerarse como de baja concordancia, ya que valores entre 0,40 a 0,75 se consideran satisfactorios ${ }^{15,16}$.

Para interpretar este resultado, habría que tener consideraciones de cuatro aspectos: el recurso humano que realiza las mediciones, los instrumentos que están siendo utilizados, el control de calidad de la información recolectada y la consistencia de la bases de datos.

En relación al primer aspecto, se detectó que los profesores muestran menor rigurosidad en la medición de los niños, al compararla con la efectuada por el equipo evaluador, por ejemplo, en la mayoría de los lugares aproximan a la unidad lo que se refleja en falta de exactitud de las medidas antropométricas tomadas. En cuanto a los instrumentos, se pudo comprobar la carencia y deficiencia de ellos en algunos lugares, es así que se comprobó la inexistencia de antropómetros en algunas escuelas y en las que había, éstos no permitían detectar diferencias milimétricas en la estatura. La mayonía de las balanzas eran poco precisas, al no ser calibradas periódicamente. La utilización de instrumentos inadecuados son factores que influyen en la deficiencia del dato recolectado.

La deficiencia en el sistema de control de calidad de los datos, tanto a nivel de la recolección como del almacenamiento, es otro problema comprobado y que es susceptible de ser mejorado. En relación a la supervisión en la recolección, ésta no era efectuada rutinariamente, lo cual impedía la corrección oportuna y, a su vez, las inconsistencias detectadas en las bases de datos reflejan la falta de un sistema de verificación del ingreso de ellos.

El tener recursos humanos capacitados y supervisados, además de instrumentos periódicamente calibrados, son requisitos para conseguir validez y precisión de las mediciones antropomé- tricas, por lo tanto, debieran destinarse recursos para la adquisición y mantención de este tipo de equipamiento, además de asegurar una supervisión permanente y organizada.

Para la realización exitosa de la medición de peso y estatura es importante, también, contar con el apoyo del profesorado responsable de estas mediciones y esto se logra a través de la comprensión de la importancia de la evaluación periódica y que estas mediciones se efectúen siguiendo normas establecidas, evitando la rotación de los profesores encargados.

La importancia de contar con mediciones confiables radica en que estas informaciones se están utilizando como sistema de vigilancia epidemiológica nutricional de este grupo poblacional a diferentes niveles (nacional, regional, comunal) y en varios países sirve para identificar a la población escolar beneficiaria de programas sociales o nutricionales y por esta vía disminuir la brecha de inequidad existente $^{17-21}$. La falta de rigurosidad en la recolección de las mediciones puede llevar a incluir beneficiarios que no les comespondería acceder a los potenciales programas (falsos positivos), con las consecuencias de generar un gasto mayor al necesanio, y por otro lado, en el caso de subestimar a los beneficiarios (falsos negativos), implicaría la no inclusión de los que realmente lo necesitan.

El hecho que la proporción de niños clasificados de bajo peso haya sido utilizada previamente como criterio para definir beneficiarios del Programa de Alimentación Escolar de Chile ${ }^{18}$, pudiera explicar, al menos en los catalogados con bajo peso, que el profesorado hubiera incorporado falsos positivos para que su establecimiento educacional reciba más beneficios. Este supuesto debiera ser investigado en profundidad.

También debe mencionarse que informes internacionales sobre la situación de salud y nutrición de los países indican que la estatura y, más específicamente, la proporción de niños con déficit de crecimiento en talla al ingreso a la escuela son indicadores de desnutrición crónica infantil que informa acerca de la historia de salud y nutrición de la población infantili ${ }^{22,23}$. Estos últimos años, al irse observando un aumento de los niveles de sobrepeso y obesidad, el tener vigilancia de los indicadores relacionados con el peso es una necesidad en todo programa destinado a controlar el exceso de peso. 
El tener un sistema de vigilancia nutricional que consigne en forma periódica el peso y la estatura de las cohortes que ingresan y permanecen en las escuelas, permite detectar precozmente a las cohortes que presentan problemas nutricionales. La finalidad de la identificación temprana de poblaciones en riesgo, tanto en déficit 0 exceso nutricional, permite intervenir de manera oportuna para evitar el surgimiento de factores de riesgo de enfermedades crónicas.

Se debe reconocer que este estudio presenta algunas limitaciones, entre las que se encuentra el haber medido a los escolares no exactamente el mismo día entre ambos grupos de evaluadores, lo que pudiera señalar que algunas diferencias observadas en las medidas antropométricas recolectadas (especialmente las de pesos) se deban en parte al efecto del crecimiento real. Aunque dudamos de esta posible subestimación, como fue mencionado en la metodología, ya que efectuamos una corrección estandarizada del valor del peso en la medida tomada por el profesorado y que los análisis estratificados entre los que tenían una variación mínima de las fechas de los exámenes y en aquellos que las fechas fueron mayores (un mes), estos análisis no mostraron diferencias significativas en los

\section{REFERENCIAS}

1. BarRía RM, Amigo H. Nutrition transition: a review of Latin American profile. Arch Latinoam Nutr 2006; 56: 3-11.

2. Vio F, Albala C, Kain J. Nutrition transition in Chile revisited: mid-term evaluation of obesity goals for the period 2000-2010. Public Health Nutr 2007; 6: 18.

3. Durán P, Cabalerero B, De Onis M. The association between stunting and overweight in Latin American and Caribbean preschool children. Food Nutr Bull 2006; 27: 300-5.

4. Botton J, Heude B, Kettaneh A, Borys JM, Lommez A, BRESSON JL ET AL; FLVS StUdy GROUP. Cardiovascular risk factor levels and their relationships with overweight and fat distribution in children: the Fleurbaix Laventie Ville Santé II study. Metabolism 2007; 56: 614-22.

5. Koenigsberg J, Boyd GS, Gidding SS, Hassink SG, FALKNER B. Association of age and sex with cardiovascular risk factors and insulin sensitivity in valores de concordancia de las medidas efectuadas en distintas fechas.

El esfuerzo que está realizando la Junta Nacional de Auxilio Escolar y Becas del Ministerio de Educación de Chile por montar, mantener y ahora mejorar el sistema de vigilancia nutricional de los escolares, que está disponible a través de la red computacional, debe ser reconocido ya que el monitoreo epidemiológico periódico aporta importantes antecedentes, no sólo para conocer la evolución de la situación nutricional de los escolares chilenos, sino puede ser utilizado para la elaboración de políticas y programas destinado a mejorar las condiciones de salud y nutrición de los escolares.

\section{Agradecimientos}

A Ivana Pivatto y Daniela Adjemian, quienes supervisaron la recolección de información y a Andrea Thiers y Pilar González por su rigurosidad y eficiencia en las mediciones de los escolares. Mención especial para la Junta Nacional de Auxilio Escolar y Becas por haber financiado el estudio y de esta manera permitir que esta investigación se realizara. Finalmente a las escuelas que permitieron el acceso a su institución para la recolección de datos antropométricos de sus escolares y a los padres de los niños que accedieron participar en el estudio.

overweight children and adolescents. J Cardiometab Syndr 2006; 1: 253-8.

6. De Onis M, Onyango AW, Van den Broeck J, Chumlea WC, Martorell R. Measurement and standardization protocols for anthropometry used in the construction of a new international growth reference. Food Nutr Bull 2004; 25 (1 Suppl): S27-36.

7. Vegelin AL, Brukx LJ, Waelkens JJ, Van den Broeck J. Influence of knowledge, training and experience of observers on the reliability of anthropometric measurements in children. Ann Hum Biol 2003; 30: 6579.

8. UlJasZek SJ, KeRR DA. Anthropometric measurement error and the assessment of nutritional status. $\mathrm{Br} \mathrm{J}$ Nutr 1999; 82: 165-77.

9. Junta Nacional de Auxilo Escolar y Becas (JUNAEB). Estudio sobre situación nutricional según criterios de obesidad, retraso de talla y desnutrición, en escolares de primero básico de establecimientos municipalizados y particulares subvencionados, según región y comuna. 2004. Disponible en: www.junaeb.cl [Consultado el 16 de mayo de 2007]. 
10. Junta Nacional de Auxilo Escolar y Becas (JUNAEB). Manual de salud del escolar. Disponible en www.junaeb.cl/manual_salud/JUNAEB.pdf [Consultado en Mayo, 2007].

11. НАBicht JP. Estandarización de métodos epidemiológicos cuantitativos sobre el terreno. Bol Oficina Sanit Panam 1974; 76: 375-84.

12. National Center for Health Statistical (NCHS) Centers for disease control and prevention (CDC) 2002 y 2000. Disponible en: http: //www.cdc.gov/ growthcharts [Consultado mayo 2007]

13. CoHEn JA. A coefficient of agreement for nominal scales. Educ Psychol Meas 1960; 20: 37-46.

14. FLEISS JL. Statistical Methods for Rates and Proportions. $2^{\text {nd }}$ ed. New York, NY: John Wiley and Sons; 1981.

15. Szkьo M, Nieto J. Epidemiología Intermedia, Conceptos y aplicaciones. Aseguramiento y control de la calidad. 298-349. Ediciones Díaz de Santos, Madrid, 2003.

16. Byrt T. How good is that agreement? Epidemiology 1996; 7: 561.
17. Morón C, Mazar I. Determinants of success in nutrition and food security programs. Arch Latinoam Nutr 2004; 54 (Suppl 1): 20-3.

18. Amgo H. School feeding programmes in Latin America. An analysis. Arch Latinoam Nutr 1997; 47: 299-304.

19. Musgrove P. Feeding Latin America's children. World Bank Res Obs 1993; 8: 23-45.

20. Devaney BL, Eluwood MR, Love JM. Programs that mitigate the effects of poverty on children. Future Child 1997; 7: 88-112.

21. LARrea C, Freire W. Social inequality and child malnutrition in four Andean countries. Rev Panam Salud Publica 2002; 11: 356-64.

22. Beaton GH, Bengoa JM. Practical population indicators of Health \& Nutrition. Anexo 3. Nutrition in preventive medicine. The mayor deficiency syndromes, epidemiology, and approaches to control. World Health Organization. Geneva. 1976.

23. FrongiLo EA JR, De Onis M, Hanson KM. Socioeconomic and demographic factors are associated with worldwide patterns of stunting and wasting of children. J Nutr 1997; 127: 2302-9. 